\title{
Review
}

Neuropsychobiology

\section{Peripheral Glutamate Levels in Schizophrenia: Evidence from a Meta-Analysis}

\author{
Jiali Song ${ }^{a}$ Andrea Viggiano ${ }^{c}$ Marcellino Monda ${ }^{d}$ Vincenzo De Luca ${ }^{b}$ \\ ${ }^{a}$ Center for Addiction and Mental Health, and ${ }^{b}$ Department of Psychiatry, University of Toronto, \\ Toronto, Ont., Canada; ' ${ }^{C}$ Department of Medicine and Surgery, University of Salerno, Salerno, and \\ ${ }^{\mathrm{d}}$ Department of Experimental Medicine, Second University of Naples, Naples, Italy
}

\section{Key Words}

Schizophrenia $\cdot$ Glutamate $\cdot$ Amino acids $\cdot$ Blood

\begin{abstract}
Background: Recent research attempting to develop novel medications has turned to glutamatergic signaling pathways to find effective treatments for symptom clusters of schizophrenia. This meta-analysis was undertaken to clarify whether a difference in peripheral glutamate levels exists between patients with schizophrenia and healthy controls. Methods: The electronic databases Ovid MEDLINE, PubMed, and PsycINFO were systematically searched up to April 2013. The search was limited to case-control studies of blood glutamate levels in schizophrenia written in English. The differences in glutamate levels were evaluated by calculating standardized mean differences (SMD). Results: We found ten studies that met the inclusion criteria for a total of 320 schizophrenia patients and 294 controls. The meta-analysis showed that peripheral glutamate levels in schizophrenia patients were significantly higher overall than in controls $(S M D=0.635, p=0.004)$. However, a significant effect of the method used to measure glutamate concentrations was found $(F=7.36, p=0.01)$ where fluorometric assay was associated with effect sizes in the opposite direction. Conclusion: A higher blood glutamate con-
\end{abstract}

\section{KARGER}

E-Mail karger@karger.com

www.karger.com/nps centration was found in patients with schizophrenia. However, given the small sample size and methodological differences among studies, this result is not conclusive. More comprehensive research is needed to understand the relationship between glutamate levels in schizophrenia in the blood and the brain.

(c) 2014 S. Karger AG, Basel

\section{Introduction}

Glutamate is the major excitatory amino acid in the mammalian central nervous system (CNS), and it is involved in most brain functions [1]. Glutamate can have a number of postsynaptic effects, which are mediated by postsynaptic receptors such as alpha-amino-3-hydroxy5-methyl-4-isoxazolepropionate (AMPA) and N-methyl-D-aspartate (NMDA). AMPA receptors play a primary role in generating excitatory postsynaptic currents and are responsible for triggering action potentials, whereas the NMDA receptor plays a critical role in synaptic plasticity [2]. The NMDA receptor is ubiquitous through the central and peripheral nervous systems [3]. It can also contribute to excitatory postsynaptic currents and dendritic spikes [2].
C 2014 S. Karger AG, Basel

0302-282X/14/0703-0133\$39.50/0
Vincenzo De Luca

Department of Psychiatry, University of Toronto 250 College Street

Toronto, ON M5T 1R8 (Canada)

E-Mail vincenzo.deluca@camh.ca 
According to the glutamatergic hypothesis of schizophrenia, the symptoms in schizophrenia are caused by a disruption in the glutamate neurotransmitter system. NMDA receptor antagonists such as ketamine are known to create symptoms similar to psychosis in healthy controls and to exacerbate positive, negative and cognitive symptoms in schizophrenia [3].

The glutamatergic hypothesis in schizophrenia has been investigated for many decades. Kim et al. [4] were among the first to investigate the glutamatergic hypothesis of schizophrenia when they reported significantly lower glutamate levels in the cerebrospinal fluid (CSF) of patients with schizophrenia than in that of healthy controls. However, later studies on blood glutamate concentrations in schizophrenia have yielded inconsistent results.

Furthermore, proton magnetic resonance spectroscopy (H-MRS) studies have found selective disturbances in the brain in schizophrenia such as higher glutamate levels in the anterior cingulate cortex (ACC) related to positive symptoms and in the parietal occipital cortex related to positive and cognitive symptoms [3,4]. Disturbances have also been found in NMDA receptor functioning in some studies, where it was found that schizophrenia patients with higher symptom severity or who were more resistant to treatment had higher and glutamate (Glx) levels than healthy controls $[5,6]$. Thus, glutamate levels may be useful for identifying potential treatment-resistant patients.

The blood-brain barrier promotes the removal of glutamate from the brain into the blood; thus very little net entry of glutamate into the brain occurs [7]. However, glutamate that exits the brain contributes significantly to the glutamate level in the blood [7]. Glutamine is a precursor to glutamate when it is partially metabolized into $\mathrm{NH}^{4+}$ and glutamate at the blood brain barrier [7] Hashimoto et al. [8] found that the glutamine/glutamate ratio was significantly elevated in the CSF of patients with schizophrenia [8]. A recent meta-analysis suggested that there may be an increased level of glutamatergic activity or an abnormality in the conversion between glutamate and glutamine in patients with schizophrenia [9].

Existing medications for the treatment of schizophrenia target positive symptoms and are ineffective for negative symptoms and cognitive deficits, leaving these symptom clusters largely untreated [10]. Recent research attempting to develop novel medications has turned to glutamatergic signaling pathways to find effective treatments for all three symptom clusters [10]. Furthermore, studies have suggested that blood glutamate concentrations may be connected with the clinical course of schizophrenia [11-13]. It has been reported that switching from conventional neuroleptics to clozapine or olanzapine significantly increased peripheral glutamate concentrations in patients $[14,15]$.

Peripheral glutamate levels may potentially serve as a biomarker in schizophrenia, which is more accessible and less costly compared to MRS. Examining disturbances in peripheral glutamate levels in schizophrenia patients could also bring insight into the course and etiology of the illness. As the results of previous studies have been inconsistent, the aim of this meta-analysis was to clarify whether peripheral glutamate levels differ between patients with schizophrenia and healthy controls.

\section{Subjects and Methods}

\section{Literature Search}

Electronic searches were done using the MEDLINE, PubMed and PsycINFO databases. Additionally, the references of relevant papers were searched manually. MEDLINE was searched up to April 2013 with the Ovid MeSH keywords 'schizophrenia' and 'glutamic acid' with the subheading 'blood' in combination with schizophrenia or glutamic acid. PubMed was searched with the keywords 'schizophrenia', 'glutamic acid' or 'glutamate', and 'blood' for any articles that were not available on MEDLINE. PsycINFO was searched up to April 2013 using the Ovid MeSH keywords 'schizophrenia' and 'glutamic acid'. The MeSH keyword 'glutamic acid' included the synonym 'glutamate'.

\section{Inclusion Criteria}

We included only articles that met the following criteria: (1) case-control study design; (2) measurement of serum or plasma glutamate in healthy controls and schizophrenia patients; (3) description of mean glutamate concentrations and their standard deviation (SD) or standard error of the mean (SEM), and (4) studies written in English.

For each included study, we collected the following information: author, year, study title, sample size, basal mean glutamate concentrations, SD or SEM of mean concentrations, component of blood from which the measurement was taken, the method of measuring glutamate concentrations, age, gender ratio, medication of patients, ethnicity, and diagnostic criteria. Only the basal glutamate concentrations from longitudinal studies were used for the purposes of this meta-analysis.

\section{Statistical Analysis}

There were two papers that divided control and case groups into subgroups; one paper separated the controls and patients by gender [16]. The means of the two control groups in the study of Tomiya et al. [16] were combined to calculate the mean of a single control group. Another paper used only one control group but separated patients into three groups: a drug-naïve group with patients who had never taken antipsychotics, a drug-free group with 
Table 1. Summary of details for the inclusion and exclusion of papers

\begin{tabular}{|c|c|c|c|c|c|c|}
\hline Study & Year & $\begin{array}{l}\text { Case-control } \\
\text { design }\end{array}$ & $\begin{array}{l}\text { Blood } \\
\text { sample }\end{array}$ & $\begin{array}{l}\text { Glutamate } \\
\text { reported }\end{array}$ & $\begin{array}{l}\text { In } \\
\text { English }\end{array}$ & Final decision \\
\hline Davis et al. [27] & 1972 & $\checkmark$ & $\checkmark$ & $\checkmark$ & $\checkmark$ & include \\
\hline Kim et al. [22] & 1982 & $\checkmark$ & $\checkmark$ & & $\checkmark$ & exclude: did not report schizophrenia patients \\
\hline Bjerkenstedt et al. [20] & 1985 & $\checkmark$ & $\sqrt{ }$ & & $\checkmark$ & exclude: did not report glutamate levels \\
\hline Macciardi et al. [19] & 1990 & $\checkmark$ & $\checkmark$ & $\checkmark$ & $\checkmark$ & include \\
\hline Altamura et al. [35] & 1993 & $\checkmark$ & $\checkmark$ & $\checkmark$ & $\checkmark$ & include \\
\hline Evins et al. [14] & 1997 & & $\checkmark$ & $\checkmark$ & $\checkmark$ & exclude: no control group \\
\hline Tortorella et al. [26] & 2001 & $\checkmark$ & $\checkmark$ & $\checkmark$ & $\checkmark$ & include \\
\hline Goff et al. [15] & 2002 & $\checkmark$ & & $\checkmark$ & $\checkmark$ & exclude: sample not blood \\
\hline van der Heijden et al. [36] & 2004 & $\checkmark$ & $\checkmark$ & $\checkmark$ & $\checkmark$ & include \\
\hline Alfredsson and Wiesel [11] & 1989 & $\checkmark$ & $\sqrt{ }$ & $\sqrt{ }$ & $\checkmark$ & include \\
\hline Maeshima et al. [13] & 2007 & & $\checkmark$ & $\checkmark$ & $\checkmark$ & exclude: no control group \\
\hline Tomiya et al. [16] & 2007 & $\checkmark$ & $\checkmark$ & $\checkmark$ & $\checkmark$ & include \\
\hline Cai et al. [12] & 2010 & $\checkmark$ & $\checkmark$ & & $\checkmark$ & exclude: did not report glutamate levels \\
\hline He et al. [39] & 2012 & $\checkmark$ & $\checkmark$ & & $\checkmark$ & exclude: did not report glutamate levels \\
\hline Ohnuma et al. [21] & 2012 & & $\checkmark$ & $\checkmark$ & $\checkmark$ & exclude: no control group \\
\hline
\end{tabular}

patients who went through a washout period and a neuroleptic group with patients who were taking neuroleptics [17]. These three groups were also combined into one group and the group mean and variance were calculated. The variance for each of the two studies was calculated using the formula:

$$
s_{w}^{2}=\frac{\left(n_{1}-1\right) s_{1}^{2}+\left(n_{2}-1\right) s_{2}^{2}+\ldots+\left(n_{k}-1\right) s_{k}^{2}}{n_{1}+n_{2}+\ldots+n_{k}-k},
$$

where $\mathrm{n}$ was the sample size, $\mathrm{s}$ was the variance, and $\mathrm{k}$ was the number of variances being combined [18].

All concentrations were converted to the unit micromole/liter and SEM values were converted to SD values using the formula: $\mathrm{SD}=\mathrm{SEM} \times \sqrt{ } \mathrm{n}$, where $\mathrm{n}$ was the sample size. The overall standardized mean difference (SMD) between cases and controls was calculated under a random model. The heterogeneity among studies was calculated and a funnel plot and the Egger regression test were used to detect publication bias. Metaregression analysis was performed on age, gender, medication (medicated or nonmedicated), sample (serum or plasma), ethnicity (white European or other), the method of measurement of glutamate concentrations (high-performance liquid chromatography, HPLC, ion exchange column chromatography, or fluorometric assay, FA), and whether participants were fasting before samples were taken. Due to the relatively high numbers obtained in 1 study [19], the meta-analysis was repeated with this study excluded.

In order to further control for possible effects of medication on glutamate levels, further analyses were done by excluding studies with drug-naïve patients or studies with only patients who were

Peripheral Glutamate Levels in Schizophrenia taking medication. Finally, to control for effects of the method of measurement on results, a meta-analysis was run on studies using HPLC to measure glutamate levels. p values smaller than 0.05 were considered significant. All statistical analyses were carried out using the metan package on STATA version 11.2.

\section{Results}

Overall, 27 articles were found in MEDLINE, 136 in PubMed and 335 in PsycINFO. A total of 20 studies on peripheral glutamate levels in schizophrenia were identified. Of these, three studies were excluded because healthy controls were not included in the study design $[13,14$, 20], three studies were excluded because mean glutamate levels were not reported $[14,21,22]$ and a further three studies were excluded because measurements were taken from the CSF rather than the blood $[8,15,23]$. In addition, one study was excluded because the full text of the paper could not be accessed [24]. Finally, ten studies met the inclusion criteria for a total of 320 schizophrenia patients and 294 healthy controls. The authors of one study were contacted; however, they were unable to provide further information. Table 1 summarizes the details for the inclusion and exclusion of papers. 
Davis et al. [27]

Altamura et al. [35]

van der Heijden et al. [36]

Palomino et al. [25]

Kim et al. [4]

Macciardi et al. [19]

Rao et al. [17]

Tortorella et al. [26]

Alfredsson and Wiesel [11]

Tomiya et al. [16]

Overall $\left(I^{2}=80.0 \%, p=0.000\right)$

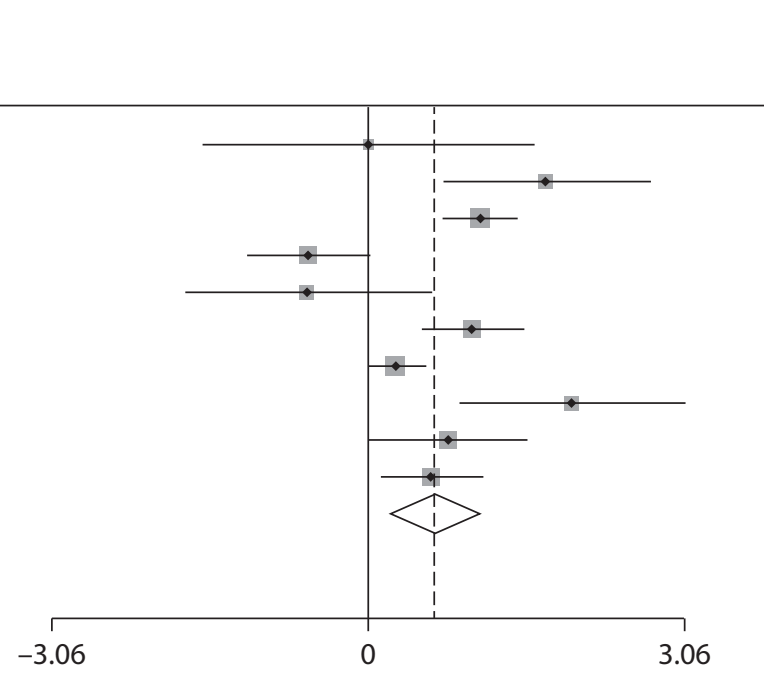

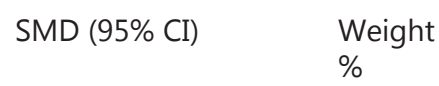

$0.00(-1.60,1.60) \quad 4.80$

$1.72(0.72,2.72) \quad 8.10$

$1.08(0.72,1.44) \quad 13.24$

$-0.58(-1.18,0.02) \quad 11.35$

$-0.58(-1.76,0.60) \quad 6.90$

$1.00(0.51,1.48) \quad 12.28$

$0.27(-0.02,0.56) \quad 13.69$

$1.98(0.89,3.06) \quad 7.50$

$0.77(0.01,1.54) \quad 9.93$

$0.61(0.12,1.10) \quad 12.23$

$0.64(0.21,1.06) \quad 100.00$

Note: Weights are from random effects analysis

Fig. 1. Forest plot with combined effects calculated for studies by Rao et al. [17] and Tomiya et al. [16]; overall effect size calculated under random model.

Table 2. Results of metaregression analysis

\begin{tabular}{lccccc}
\hline Covariate & $\mathrm{n}$ & $\beta$ & $\mathrm{SE}$ & $\mathrm{p}$ & Intercept \\
\hline Age & 5 & 0.04 & 0.09 & 0.67 & -0.65 \\
Gender & 7 & -0.001 & 0.02 & 0.95 & 0.90 \\
Ethnicity & 10 & -0.03 & 0.86 & 0.97 & 0.64 \\
Medication & 10 & -0.41 & 0.53 & 0.46 & 0.81 \\
Sample type & 10 & -0.09 & 0.56 & 0.86 & 0.67 \\
HPLC & 10 & 1.00 & 0.40 & 0.03 & 0.11 \\
IECC & 10 & -0.19 & 0.60 & 0.75 & 0.69 \\
FA & 10 & -1.44 & 0.44 & 0.01 & 0.86 \\
Fasting & 10 & -0.40 & 0.58 & 0.51 & 0.75 \\
\hline
\end{tabular}

IECC $=$ Ion exchange column chromatography.

Of note, the study of Tomiya et al. [16] reported glutamate levels in males and females separately. To further understand the source of heterogeneity in our studies, we analyzed males and females of this study as two individual samples. We also combined the males and females into one sample, as described in Methods. We found significant heterogeneity among studies in both cases, i.e. when we considered the males and females as separate samples $(\mathrm{Q}=48.02$, d.f. $=10, \mathrm{p}<0.001)$ as well as when we combined the males and females of the study into one sample
$(\mathrm{Q}=45.07$, d.f. $=9, \mathrm{p}<0.001)$. The random effects model meta-analysis (fig. 1) revealed significantly higher levels of glutamate in patients with schizophrenia than in healthy controls in both models, i.e. where males and females were separate samples $(\mathrm{SMD}=0.652$, d.f. $=10, \mathrm{p}=0.001)$ and when they were a combined sample $(\mathrm{SMD}=0.635$, d.f. $=$ $9,95 \% \mathrm{CI}=0.20-1.06, \mathrm{p}=0.004)$. The fixed effects model meta-analysis confirmed this result $(\mathrm{p}<0.001)$.

We tested the publication bias to see whether smaller studies were showing higher differences and larger studies showing smaller differences. There was no evidence that publication bias was present in our sample. The funnel plot, as shown in figure 2, was symmetrical and the Egger test, graphed in figure 3, was not significant (intercept $=0.40, \mathrm{p}=0.806,95 \% \mathrm{CI}=-3.28$ to 4.09 ), confirming that significant publication bias was not present.

Metaregression analysis was performed to control for covariates, the results of which are summarized in table 2 . The metaregressions revealed no significant association between differences in glutamate levels and age $(\mathrm{SE}=0.09$, d.f. $=4, \mathrm{p}=0.67)$, gender $(\mathrm{SE}=0.02$, d.f. $=6, \mathrm{p}=0.95)$, ethnicity $(\mathrm{SE}=0.86$, d.f. $=9, \mathrm{p}=0.97)$, medication $(\mathrm{SE}=$ 0.53 , d.f. $=9, p=0.746$ ), sample type (serum versus plasma) $(\mathrm{SE}=0.56$, d.f. $=9, \mathrm{p}=0.86)$, and whether patients were fasting ( $\mathrm{SE}=0.58$, d.f. $=9, \mathrm{p}=0.51)$. However, the method used for measuring glutamate levels was a significant covariate $(\mathrm{F}=7.36, \mathrm{p}=0.01)$. In particular, studies 


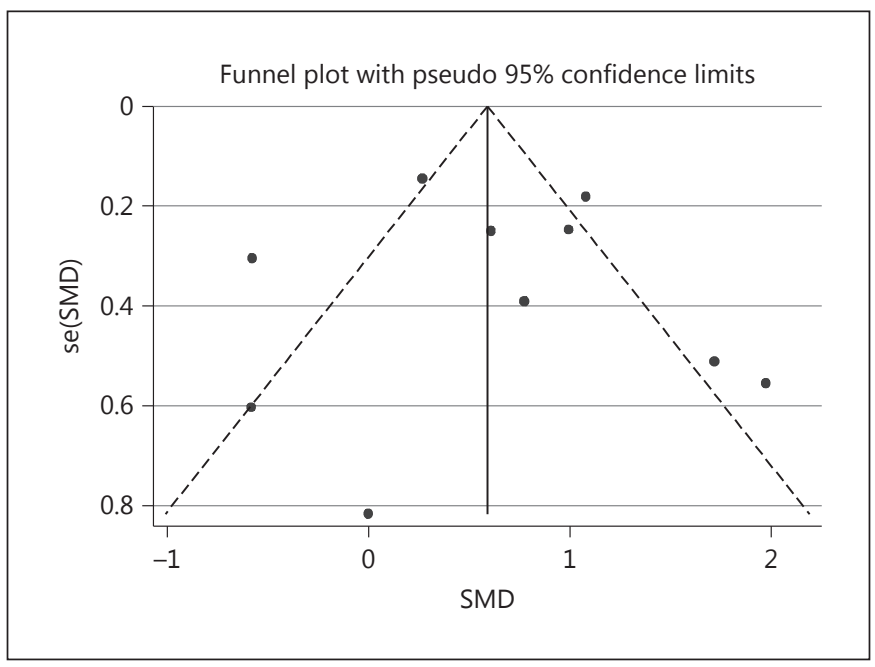

Fig. 2. Funnel plot showing no significant asymmetry and no significant publication bias.

using HPLC produced greater differences between patients and controls. On the other hand, FA produced effect sizes in the opposite direction.

After excluding the study by Macciardi et al. [19], the random effects meta-analysis still revealed a higher serum glutamate concentration in schizophrenia patients than healthy controls $(\mathrm{SMD}=0.59$, d.f. $=8,95 \% \mathrm{CI}=0.10-1.01$, $\mathrm{p}<0.001)$. The analysis which excluded studies with drugnaïve patients showed higher glutamate levels in schizophrenia patients than controls $(\mathrm{SMD}=0.90$, d.f. $=7,95 \%$ $\mathrm{CI}=0.51-1.283, \mathrm{p}<0.001)$. The meta-analysis which excluded studies with patients on medication showed a similar effect (SMD $=0.82$, d.f. $=5,95 \% \mathrm{CI}=0.04-1.60, \mathrm{p}=$ 0.038 ). Finally, an analysis was run only on studies using HPLC to measure glutamate levels, which still showed an effect in the same direction $(\mathrm{SMD}=1.075$, d.f. $=4,95 \%$ $\mathrm{CI}=0.66-1.48, \mathrm{p}<0.001)$.

\section{Discussion}

Our results showed a significant difference in peripheral glutamate levels between patients with schizophrenia and healthy controls. Based on the results of the metaregression analysis, this difference in glutamate level seems to be independent from age, gender, medication, and ethnicity of the patients. The reference peripheral glutamate concentration is $50-100 \mu \mathrm{mol} / \mathrm{l}$ [7]. However, in the 10 studies that were included, the ranges for the mean glutamate levels after conversion for controls and patients

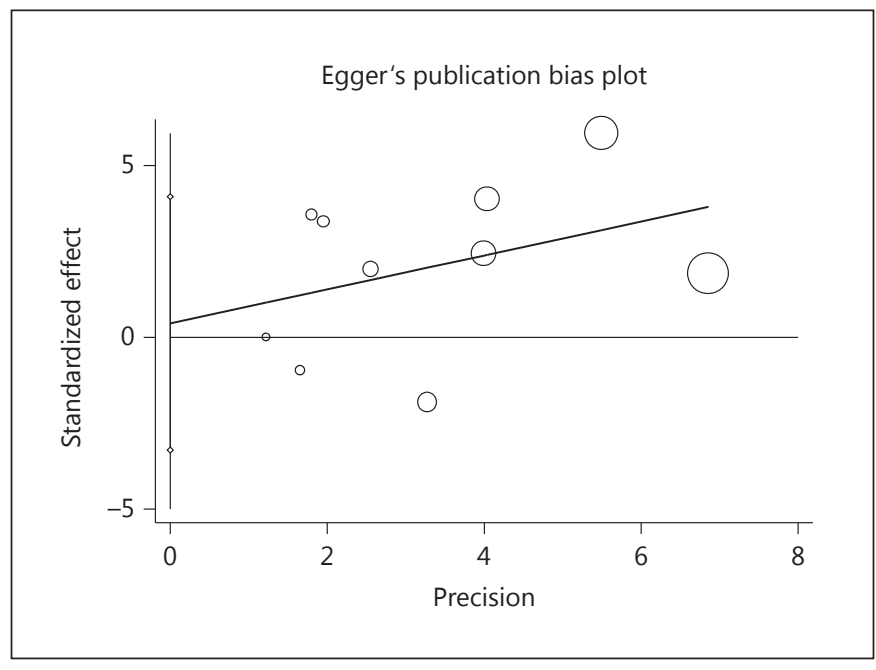

Fig. 3. Egger's publication bias plot; the size of the circles represents the weight of each study from random effects analysis.

were $19.5-28,000$ and $33.4-57,000 \mu \mathrm{mol} / \mathrm{l}$, respectively. The results of many studies did not fall within the range of $50-100 \mu \mathrm{mol} / \mathrm{l}$ at all (table 3 ). It is likely that the study reporting 28,000 and $57,000 \mu \mathrm{mol} / \mathrm{l}$ had a typographical error [19]. However, even after excluding this study, patients still have significantly higher glutamate levels than controls, although the standard mean difference is slightly smaller. Thus, this effect is not due to that aforementioned study, which had the largest effect size in our sample.

In addition, the method of measuring glutamate was a significant covariate in our analysis. The correlation of HPLC with a larger effect size may suggest that HPLC is a more sensitive measure of glutamate concentration in the blood and may account for some of the large variation between studies. The FA method was correlated with an effect size in the opposite direction. The FA method measures the concentration NADPH produced from the oxidation reaction between the enzyme glutamate dehydrogenase and glutamate in the presence of $\mathrm{NADP}^{+}[25]$. It is an indirect measurement of glutamate concentration. The opposite effect sizes may have been confounded by NADPH from other sources apart from the oxidation of glutamate. This correlation could suggest a general disturbance in the glutamatergic system in patients with schizophrenia. HPLC is the more accurate method for determining the concentration of glutamate in the blood. Another possible source of heterogeneity is hemolysis, which may have released glutamate from red blood cells into the sample. Prolonged storage may also result in the 
Table 3. Study data

\begin{tabular}{|c|c|c|c|c|c|c|}
\hline \multirow[t]{2}{*}{ Study } & \multirow[t]{2}{*}{ Year } & \multicolumn{2}{|c|}{ Sample size } & \multicolumn{2}{|c|}{ Glutamate, $\mu \mathrm{mol} / \mathrm{l}$} & \multirow{2}{*}{$\begin{array}{l}\text { Original } \\
\text { units }\end{array}$} \\
\hline & & control & patient & control & patient & \\
\hline Davis et al. [27] & 1972 & 6 & 2 & $35 \pm 5.3$ & $35 \pm 7.0$ & $\mu \mathrm{mol} / 100 \mathrm{ml}$ \\
\hline Altamura et al. [35] & 1993 & 9 & 13 & $21.58 \pm 7.61$ & $47.16 \pm 18.18$ & $\mathrm{nmol} / \mathrm{ml}$ \\
\hline van der Heijden et al. [36] & 2004 & 73 & 66 & $33 \pm 12.8$ & $55.8 \pm 27.5$ & $\mu \mathrm{mol} / 1$ \\
\hline Palomino et al. [25] & 2007 & 21 & 24 & $53.39 \pm 14.25$ & $45 \pm 14.71$ & $\mu \mathrm{mol} / 1$ \\
\hline Kim et al. [4] & 1980 & 10 & 4 & $203.15 \pm 28.68$ & $188.53 \pm 7.42$ & $\mathrm{nmol} / \mathrm{ml}$ \\
\hline Macciardi et al. [19] & 1990 & 32 & 43 & $28,000 \pm 12,000$ & $57,000 \pm 37,000$ & $\mu \mathrm{mol} / \mathrm{ml}$ \\
\hline Rao et al. [17] & 1990 & 88 & 103 & $71 \pm 60$ & $85.61 \pm 47.89^{1}$ & $\mu \mathrm{mol} / 1$ \\
\hline Tortorella et al. [26] & 2001 & 10 & 10 & $38.8 \pm 18.6$ & $205.3 \pm 117.7$ & $\mu \mathrm{mol} / \mathrm{l}$ \\
\hline Alfredsson and Wiesel [11] & 1989 & 10 & 23 & $19.5 \pm 5.8$ & $33.4 \pm 21$ & $\mathrm{nmol} / \mathrm{ml}$ \\
\hline Tomiya et al. [16] & 2007 & 35 & 32 & $68.26 \pm 29.6^{2}$ & $91.3 \pm 45.1^{2}$ & $\mu \mathrm{mol} / \mathrm{l}$ \\
\hline
\end{tabular}

Values for glutamate are presented as means \pm SD. ${ }^{1}$ Combined drug-naïve, drug-free and neuroleptic-treated patient groups. ${ }^{2}$ Male and female groups combined and treated as one sample.

enzymatic conversion of glutamine into glutamate, which has been cited as a source of variability in samples [26].

In addition, we found significantly higher peripheral glutamate concentrations in patients with schizophrenia even when the analysis was repeated excluding studies with drug-naïve patients or with patients on medication. This further suggests that this difference is robust despite different effects of medication. Furthermore, the metaregression analysis revealed no evidence for an association between medication and effect size.

However, there is still reason not to overlook the potential effects of antipsychotic medication on peripheral glutamate levels. Previous research using H-MRS has found reduced central glutamate levels in some patients after antipsychotic treatment but not in others [6]. This suggests that there are individual differences in the fluctuation of glutamate levels in response to antipsychotic treatment.

In addition, medications that patients were taking before the washout period may have had an effect on glutamate levels. The length of the washout period may not have been sufficient for the patients to return to baseline. Davis et al. [27] examined the effects of tranylcypromine and L-cysteine on the plasma levels of amino acids in patients with schizophrenia and reported that glutamate concentrations had not returned to baseline 3 weeks after the patients had been taken off the medication, suggesting that washout periods of less than 3 weeks may not be enough time for the effects of the medication to disappear.
Moreover, few studies have examined peripheral glutamate levels in drug-naïve patients. Two studies included in this meta-analysis included drug-naïve patients, but they reported inconsistent results $[17,25]$. Thus, in the interpretation of our findings, more research on how the peripheral glutamate levels differ between drug-naïve and medicated patients is required to further understand the relationship and effects of medication differences.

The relationship between central and peripheral glutamate levels is not clear based on the current literature. A study on the correlation between glutamate concentrations measured in the medial prefrontal cortex using MRS and peripheral glutamate levels did not find a correlation between the two [28]. A caveat of the study is that it took measurements from the medial prefrontal cortex, so it is possible that other parts of the brain may contribute to the glutamate levels in the blood. We are not aware of studies comparing glutamate levels in other areas of the brain with peripheral levels. More research is needed to elucidate this relationship. Thus, the difference in glutamate we found may have been due to another factor which differentiates patients of schizophrenia and healthy controls, as discussed below.

The difference in glutamate levels between schizophrenia patients and healthy controls could be an effect of diet. It is known that abnormal amino acid levels are present in patients with schizophrenia [29]. It has been reported that peripheral amino acid levels can inform the CNS about the amino acid content of diet and consequently affect dietary intake [30]. Dietary behavior in pa- 
Table 4. Demographic information

\begin{tabular}{|c|c|c|c|c|c|c|c|c|}
\hline Altamura et al. [35] & $36.55 \pm 8.3$ & $32.23 \pm 13.41$ & 22.2 & 23 & washout: 3 weeks & plasma & HPLC & Italian \\
\hline van der Heijden et al. [36] & - & $32.5 \pm 13.4$ & - & 36.3 & washout: $2-9$ days & plasma & HPLC & Finnish \\
\hline Macciardi et al. [19] & - & - & 50 & 34.8 & neuroleptics & serum & IECC & Italian \\
\hline Rao et al. [17] & $25 \pm 5$ & $33 \pm 11$ & 45.5 & 45 & mixed & serum & IECC & German \\
\hline Tortorella et al. [26] & $29.6 \pm 7.8$ & $24.5 \pm 4.7$ & 40 & 40 & washout: 3 weeks & serum & HPLC & Italian \\
\hline Alfredsson and Wiesel [11] & - & $28.6 \pm 5.2$ & 50 & 39.1 & drug-naïve and washout & serum & HPLC & Swedish \\
\hline Tomiya et al. [16] & $37.8 \pm 15.15$ & $41.7 \pm 14.42$ & 40 & 40.6 & clozapine & serum & HPLC & Japanese \\
\hline
\end{tabular}

Values for age are presented as means \pm SD. IECC $=$ Ion exchange column chromatography.

tients with schizophrenia may differ from that of controls through mediators in the CNS, which may contribute to the increased peripheral glutamate levels in schizophrenia patients. Indeed, studies have found that the diets of schizophrenia patients are less healthy compared to the general population, consuming less fruit and vegetables or more calories than the general population [31].

Furthermore, the effect of antipsychotics on feeding behavior in mice has been reported. Clozapine, olanzapine, risperidone, chlorpromazine, haloperidol, and low doses of sulpiride were found to induce increased feeding behavior of palatable foods in female mice [32]. A study on the effect of olanzapine on feeding behavior in mice suggested that olanzapine reduces satiety [33]. A similar effect has been reported in humans. Patients on secondgeneration antipsychotics were found to be more responsive to external cues for eating and they felt less satiated after a meal than patients who were not on second-generation antipsychotics [34]. In this meta-analysis, only four out of the 13 studies put patients on a controlled diet, thus dietary differences between patient and controls groups should be taken into consideration $[17,26,27,35]$.

A number of papers included in this meta-analysis did not provide some of the demographic data such as the age and gender ratio of the patient and/or control groups (table 4). With this information unavailable, it is difficult to make conclusions about the samples in studies.

As the studies included in this analysis span a long range of time [1972-2007], the diagnostic criteria of schizophrenia have changed over this time, and the differences in cri- teria could have been a source of heterogeneity between studies. Overall, 3 studies used the diagnostic criteria of the DSM-III-R [11, 19, 35], 2 studies used the diagnostic criteria of the DSM-IV $[25,36]$ and 1 study used the diagnostic criteria of the Research Diagnostic Criteria by Schneider [17]. Schneider's Research Diagnostic Criteria is only available in German and could not be reviewed for this analysis; four studies did not report the diagnostic criteria used [4, $16,26,27]$. The criteria of the DSM-III-R are different from those of the DSM-IV. The DSM-IV includes more negative symptoms and places more emphasis on the chronic nature of schizophrenia than the DSM-III [37]. However, Roberts et al. [38] reported high concordance rates between schizophrenia diagnoses according to the DSM-III$\mathrm{R}$ and the DSM-IV. Thus, the differing diagnostic criteria are not likely to be a factor in the variance.

The significant heterogeneity of the studies included in our analysis is in agreement with the heterogeneity found in schizophrenia. This could also be due to the small sample sizes of studies included in this meta-analysis, as shown in table 3. Particularly, the studies by Davis et al. [27] and Kim et al. [4] had only 2 and 4 patients, respectively. Neither study found differences between patients and controls, so the sample size may have been a factor in masking any differences. In addition, this analysis included only ten studies and the pooled sample size was 294 controls and 320 patients. However, this could also be an indication of a robust effect. A bigger and more comprehensive sample would produce more conclusive findings. 


\section{Conclusion}

Our finding that schizophrenia patients have higher peripheral glutamate levels than healthy controls presents further evidence for the involvement of the glutamatergic system in schizophrenia. However, the sample size of this analysis was small, and the methods used to measure glutamate levels varied between studies, which may have had an effect on the results of the studies as revealed by the metaregression analysis. A larger and more comprehensive sample size with more standard- ized methods of quantification would provide more conclusive results. The effects of antipsychotic medications and the relationship between central and peripheral glutamate levels need further investigation before more definitive conclusions can be drawn. It would be of clinical value to determine whether glutamate can be used as a clinical marker in schizophrenia. However, further research is required with regard to antipsychotic medication and its effects on the glutamatergic signaling system as well as the relationship between central and peripheral glutamate levels.

\section{References}

1 Danbolt NC: Glutamate uptake. Prog Neuro- 11 Alfredsson G, Wiesel FA: Monoamine metabbiol 2001;65:1-105.

$>2$ Coyle JT: Glutamate and schizophrenia: beyond the dopamine hypothesis. Cell Mol Neurobiol 2006;26:363-382.

3 Coyle JT, Basu A, Benneyworth M, Balu D, Konopaske G: Glutamatergic synaptic dysregulation in schizophrenia: therapeutic implications. Handb Exp Pharmacol 2012;213: 267-295.

-4 Kim JS, Kornhuber HH, Holzmuller B, Schmid-Burgk W, Mergner T, Krzepinski G: Reduction of cerebrospinal fluid glutamic acid in Huntington's chorea and in schizophrenic patients. Arch Psychiatr Nervenkr 1980;228:7-10

5 Merritt K, McGuire P, Egerton A: Relationship between glutamate dysfunction and symptoms and cognitive function in psychosis. Front Psychiatry 2013;4:151.

6 Poels EM, Kegeles LS, Kantrowitz JT, Javitt DC, Lieberman JA, Abi-Dargham A, Girgis RR: Glutamatergic abnormalities in schizophrenia: a review of proton MRS findings. Schizophr Res 2014;152:325-332.

7 Hawkins RA: The blood-brain barrier and glutamate. Am J Clin Nutr 2009;90:867S874 S.

$>8$ Hashimoto K, Engberg G, Shimizu E, Nordin C, Lindstrom LH, Iyo M: Elevated glutamine/ glutamate ratio in cerebrospinal fluid of first episode and drug naive schizophrenic patients. BMC Psychiatry 2005;5:6

>9 Marsman A, van den Heuvel MP, Klomp DW Kahn RS, Luijten PR, Hulshoff Pol HE: Glutamate in schizophrenia: a focused review and meta-analysis of ${ }^{1} \mathrm{H}-\mathrm{MRS}$ studies. Schizophr Bull 2013;39:120-129.

10 Noetzel MJ, Jones CK, Conn PJ: Emerging approaches for treatment of schizophrenia: modulation of glutamatergic signaling. Discov Med 2012;14:335-343. olites and amino acids in serum from schizophrenic patients before and during sulpiride treatment. Psychopharmacology (Berl) 1989; 99:322-327.

2 Cai HL, Zhu RH, Li HD, Zhang XH, Hu L, Yang W, et al: Elevated plasma gamma-aminobutyrate/glutamate ratio and responses to risperidone antipsychotic treatment in schizophrenia. Prog Neuropsychopharmacol Biol Psychiatry 2010;34:1273-1278.

13 Maeshima H, Ohnuma T, Sakai Y, Shibata N Baba $\mathrm{H}$, Ihara $\mathrm{H}$, et al: Increased plasma glutamate by antipsychotic medication and its relationship to glutaminase 1 and 2 genotypes in schizophrenia - Juntendo University Schizophrenia Projects (JUSP). Prog Neuropsychopharmacol Biol Psychiatry 2007;31: 1410-1418.

14 Evins AE, Amico ET, Shih V, Goff DC: Clozapine treatment increases serum glutamate and aspartate compared to conventional neuroleptics. J Neural Transm 1997;104:761-766.

15 Goff DC, Hennen J, Lyoo IK, Tsai G, Wald LL, Evins AE, et al: Modulation of brain and serum glutamatergic concentrations following a switch from conventional neuroleptics to olanzapine. Biol Psychiatry 2002;51:493-497.

16 Tomiya M, Fukushima T, Watanabe H, Fukami G, Fujisaki M, Iyo M, et al: Alterations in serum amino acid concentrations in male and female schizophrenic patients. Clin Chim Acta 2007;380:186-190.

17 Rao ML, Gross G, Strebel B, Braunig P, Huber G, Klosterkotter J: Serum amino acids, central monoamines, and hormones in drug-naive, drug-free, and neuroleptic-treated schizophrenic patients and healthy subjects. Psychiatry Res 1990;34:243-257.

18 Headrick TC: Statistical Simulation: Power Method Polynomials and Other Transformations. Boca Raton, Chapman \& Hall/CRC, 2010.

19 Macciardi F, Lucca A, Catalano M, Marino C, Zanardi R, Smeraldi E. Amino acid patterns in schizophrenia: some new findings. Psychiatry Res 1990;32:63-70.
20 Bjerkenstedt L, Edman G, Hagenfeldt L, Sedvall $\mathrm{G}$, Wiesel FA: Plasma amino acids in relation to cerebrospinal fluid monoamine metabolites in schizophrenic patients and healthy controls. Br J Psychiatry 1985;147:276-282.

21 Ohnuma T, Sakai Y, Maeshima H, Higa M, Hanzawa R, Kitazawa M, et al: No correlation between plasma NMDA-related glutamatergic amino acid levels and cognitive function in medicated patients with schizophrenia. Int J Psychiatry Med 2012;44:17-27.

22 Kim JS, Schmid-Burgk W, Claus D, Kornhuber $\mathrm{HH}$ : Increased serum glutamate in depressed patients. Arch Psychiatr Nervenkr 1982;232:299-304

23 Bendikov I, Nadri C, Amar S, Panizzutti R, De Miranda J, Wolosker H, Agam G: A CSF and postmortem brain study of D-serine metabolic parameters in schizophrenia. Schizophr Res 2007;90:41-51.

24 Morshed NM, Sobhan MA, Nahar JS, Keramat Ali SM, Shams M: Excitatory amino acid neurotransmitters in schizophrenia. Bangladesh Med Res Counc Bull 2005;31: 15-20.

25 Palomino A, Gonzalez-Pinto A, Aldama A, Gonzalez-Gomez C, Mosquera F, GonzalezGarcia G, et al: Decreased levels of plasma glutamate in patients with first-episode schizophrenia and bipolar disorder. Schizophr Res 2007;95:174-178.

26 Tortorella A, Monteleone P, Fabrazzo M, Viggiano A, De Luca L, Maj M: Plasma concentrations of amino acids in chronic schizophrenics treated with clozapine. Neuropsychobiology 2001;44:167-171.

27 Davis JM, Spaide JK, Himwich HE: Effects of tranylcypromine and L-cysteine on plasma amino acids in controls and schizophrenic patients. Am J Clin Nutr 1972;25:302-310.

28 Shulman Y, Grant S, Seres P, Hanstock C, Baker G, Tibbo P: The relation between peripheral and central glutamate and glutamine in healthy male volunteers. J Psychiatry Neurosci 2006;31:406. 
29 De Luca V, Viggiano E, Messina G, Viggiano A, Borlido C, Viggiano A, et al: Peripheral amino acid levels in schizophrenia and antipsychotic treatment. Psychiatry Investig 2008;5:203-208.

30 Monda M, Sullo A, De Luca V, Pellicano MP Viggiano A: L-threonine injection into PPC modifies food intake, lateral hypothalamic activity, and sympathetic discharge. Am J Physiol 1997;273:R554-R559.

-31 Bushe C, Haddad P, Peveler R, Pendlebury J: The role of lifestyle interventions and weight management in schizophrenia. J Psychopharmacol 2005;19(suppl):28-35.

32 Kaur G, Kulkarni SK: Studies on modulation of feeding behavior by atypical antipsychotics in female mice. Prog Neuropsychopharmacol Biol Psychiatry 2002;26:277-285.
33 Thornton-Jones Z, Neill JC, Reynolds GP: The atypical antipsychotic olanzapine enhances ingestive behaviour in the rat: a preliminary study. J Psychopharmacol 2002;16: 35-37.

34 Blouin M, Tremblay A, Jalbert ME, Venables $\mathrm{H}$, Bouchard RH, Roy MA, et al: Adiposity and eating behaviors in patients under second generation antipsychotics. Obesity (Silver Spring) 2008;16:1780-1787.

35 Altamura CA, Mauri MC, Ferrara A, Moro AR, D’Andrea G, Zamberlan F: Plasma and platelet excitatory amino acids in psychiatric disorders. Am J Psychiatry 1993;150:17311733 .
36 van der Heijden FM, Tuinier S, Fekkes D, Sijben AE, Kahn RS, Verhoeven WM: Atypical antipsychotics and the relevance of glutamate and serotonin. Eur Neuropsychopharmacol 2004;14:259-265.

37 McGlashan TH: The DSM-IV version of schizophrenia may be harmful to patients' health. Early Interv Psychiatry 2007;1:289293.

38 Roberts SB, Hill CA, Dean B, Keks NA, Opeskin K, Copolov DL: Confirmation of the diagnosis of schizophrenia after death using DSM-IV: a Victorian experience. Aust NZ J Psychiatry 1998;32:73-76.

-39 He Y, Yu Z, Giegling I, Xie L, Hartmann AM, Prehn C, Adamski J, Kahn R, Li Y, Illig T, Wang-Sattler R, Rujescu D: Schizophrenia shows a unique metabolomics signature in plasma. Transl Psychiatry 2012;2:e149. 